\title{
Noninvasive blood glucose monitoring
}

National Diabetes Information Clearinghouse (NDIC)

\section{Definitions}

Blood glucose

Defined by National Diabetes Information Clearinghouse (NDIC)

\section{Source}

National Diabetes Information Clearinghouse (U.S.). (2009). The diabetes dictionary. [Bethesda, Md.]: U.S. Dept. of Health and Human Services, National Institutes of Health, National Institute of Diabetes and Digestive and Kidney Diseases, National Diabetes Information Clearinghouse.

Measuring blood glucose without pricking the finger to obtain a blood sample. 